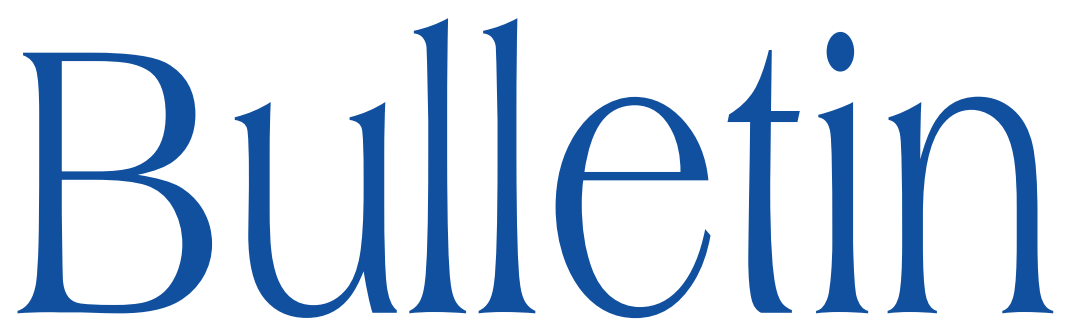

de la SOCIÉTÉ MATHÉMATIQUE DE FRANCE

\title{
BIRATIONAL GEOMETRY OF QUADRICS
}

\section{Burt Totaro}

\section{Tome 137}

Fascicule 2

2000 


\title{
BIRATIONAL GEOMETRY OF QUADRICS
}

\author{
BY BurT TOTARO
}

\begin{abstract}
We construct new birational maps between quadrics over a field. The maps apply to several types of quadratic forms, including Pfister neighbors, neighbors of multiples of a Pfister form, and half-neighbors. One application is to determine which quadrics over a field are ruled (that is, birational to the projective line times some variety) in a larger range of dimensions. We describe ruledness completely for quadratic forms of odd dimension at most 17 , even dimension at most 10 , or dimension 14 . The proof uses a new structure theorem for 14-dimensional forms, generalizing Izhboldin's theorem on 10-dimensional forms. We also show that Vishik's 16-dimensional form is ruled.
\end{abstract}

RÉSumÉ (La géométrie birationnelle des quadriques). - Nous construisons de nouvelles applications birationnelles entre quadriques sur un corps. Diverses formes quadratiques sont considérées: les voisines de Pfister, les voisines des multiples d'une forme de Pfister, et les demi-voisines. Un corollaire est la détermination des quadriques réglées (c'est-à-dire birationnelles au produit de la droite projective et d'une variété) en certaines dimensions. Nous décrivons complètement les quadriques réglées lorsque la forme quadratique est de dimension impaire inférieure à 17 , de dimension paire inférieure à 10 , ou de dimension 14 . La preuve utilise un nouveau théorème de structure sur les formes de dimension 14, généralisant le théorème d'Izhboldin sur les formes de dimension 10. Nous montrons également que la forme de Vishik de dimension 16 est réglée.

Texte reçu le 2 avril 2008, accepté le 19 juin 2008

Burt Totaro, DPMMS, Wilberforce Road, Cambridge CB3 0WB, England E-mail : b.totaro@dpmms.cam.ac.uk 2000 Mathematics Subject Classification. - 11E04, $14 \mathrm{E} 05$.

Key words and phrases. — Quadratic forms, ruled varieties, birational geometry, quadratic Zariski problem. 
A central method in the theory of quadratic forms is the study of function fields of projective quadrics. In particular, it is important to ask when there is a rational map from one quadric over a field to another. This suggests the problem of determining when two quadrics are birational, which turns out to be much harder. The answer is known for quadratic forms of dimension at most 7 (thus for projective quadrics of dimension at most 5), by Ahmad-Ohm and Roussey among others [16, 1].

In particular, there is a conjectural characterization of which quadratic forms are ruled (meaning that the associated quadric is birational to $\mathbf{P}^{1}$ times some variety), Conjecture 1.1. The conjecture was known for quadratic forms of dimension at most 9 [17]. One result of this paper is to prove Conjecture 1.1 for odd-dimensional forms of dimension at most 17, and also for forms of dimension 10 or 14 (Theorem 4.1). We use in particular a new structure theorem on 14-dimensional forms (Theorem 4.2), generalizing Izhboldin's theorem on 10dimensional forms. Vishik gave an example of a 16-dimensional form with first Witt index 2 which is not divisible by a binary form; such a form should be ruled by the conjecture, but it fell outside previously known classes of ruled forms. Nonetheless, we give a new construction which shows that Vishik's form is ruled (Theorem 7.2).

In this paper we also solve the problem of birational classification for a significant class of quadratic forms, Pfister neighbors of dimension at most 16 (Corollary 3.2). (Among these forms, only the case of "special Pfister neighbors" was known before, which fails to include all Pfister neighbors in dimensions 9, 10, and 11.) We also give several generalizations for a broader class of quadratic forms: neighbors of multiples of a Pfister form (Theorems 3.1 and 6.3), strengthening Roussey's theorem in this direction. We also give the first construction of a birational map between two non-isomorphic half-neighbors in section 5 . This is genuinely different from any previously constructed birational map between quadrics.

Thanks to Alexander Vishik for allowing me to include his example of a 16-dimensional form with splitting pattern $(2,2,2,2)$ which is not divisible by a binary form (Lemma 7.1).

\section{Known low-dimensional results}

Throughout we work over fields of characteristic not 2 .

We say that two varieties over a field $k$ are birational if they are birational over $k$. In particular, a quadric (meaning a smooth projective quadric hypersurface) over $k$ is rational (birational to projective space over $k$ ) if and only if it is isotropic, meaning that it has a $k$-rational point. The proof is standard (stereographic projection). So the nontrivial problems of birational geometry

TOME $137-2009-\mathrm{N}^{\circ} 2$ 
for quadrics are concerned only with anisotropic quadrics, and we often tacitly assume that the quadrics we consider are anisotropic. In particular, the whole subject would be vacuous over an algebraically closed field.

A quadratic form $q$ (always assumed nondegenerate) on an $n$-dimensional vector space over $k$ determines a projective quadric $Q=\{q=0\}$ of dimension $n-2$ in $\mathbf{P}^{n-1}$. The function field $k(q)$ means the field $k(Q)$ of rational functions on $Q$. We say that two quadratic forms $q_{1}$ and $q_{2}$ are similar if $q_{1}$ is isomorphic to $a q_{2}$ for some $a$ in $k^{*}$. Two forms are similar if and only if the corresponding projective quadrics are isomorphic. The group of similarity factors $G(q)$ is the group of elements $a$ in $k^{*}$ with $q$ isomorphic to aq. Every form can be written as a diagonal form $\left\langle a_{1}, \ldots, a_{n}\right\rangle=a_{1} x_{1}^{2}+\cdots+a_{n} x_{n}^{2}$ for some $a_{i} \in k^{*}$. The discriminant $\operatorname{det}_{ \pm} q$ of a quadratic form $q=\left\langle a_{1}, \ldots, a_{n}\right\rangle$ is $(-1)^{n(n-1) / 2} a_{1} \cdots a_{n}$ in $\left(k^{*}\right) /\left(k^{*}\right)^{2}=H^{1}\left(k, \mathbf{F}_{2}\right)$.

We recall the definition of the splitting pattern of a quadratic form $q$. Let $k_{0}=k, q_{0}=q_{\text {an }}$ (the anisotropic part of $q$, well-defined up to isomorphism), and then inductively define $k_{j}=k_{j-1}\left(q_{j-1}\right)$ and $q_{j}=\left(\left(q_{j-1}\right)_{k_{j}}\right)_{\text {an }}$. We stop when $q_{h}$ has dimension at most 1 . Then the splitting pattern $\mathbf{i}(q)$ is the sequence $\left(i_{1}, \ldots, i_{h}\right)$ where $i_{j}$ is the Witt index $i_{W}\left(\left(q_{j-1}\right)_{k_{j}}\right)$. The splitting tower of $q$ is the sequence of fields $k_{0}, \ldots, k_{h}$. For the associated projective quadric $Q$, we write $i_{j}(Q)=i_{j}(q)$.

We say that two varieties over a field $k$, possibly of different dimensions, are stably birational if their products with some projective spaces over $k$ are birational. For quadrics $X$ and $Y$, a simple argument shows that stable birational equivalence is equivalent to the apparently weaker condition that $X$ is isotropic over the function field of $Y$ and vice versa, or equivalently that there are rational maps from $X$ to $Y$ and from $Y$ to $X$ over $k$ [15, Theorem X.4.25]. We say that two quadratic forms over $k$ are birational if the associated projective quadrics are birational.

By Karpenko and Merkurjev, we know exactly when an anisotropic quadric $X$ is stably birational to a variety of lower dimension; this happens if and only if the first Witt index $i_{1}(X)$ is greater than 1 [10, Theorem 3.1]. (For the quadric $X$ associated to an anisotropic quadratic form $q$, the first Witt index $i_{1}(X)$ is the maximal dimension of an isotropic linear subspace for $q$ over the function field $k(X)$.) Every quadric $X$ is stably birational to any subquadric $Y$ of codimension at most $i_{1}(X)-1$. This suggests the following conjecture, which would imply in particular that an anisotropic quadric is ruled (birational to $Y \times \mathbf{P}^{1}$ for some variety $Y$ over $k$ ) if and only if its first Witt index is greater than 1 . 
Conjecture 1.1 (Ruledness conjecture). - Let $X$ be an anisotropic quadric over a field. Then $X$ is birational to $Y \times \mathbf{P}^{i_{1}(X)-1}$ for some subquadric $Y$ of codimension $i_{1}(X)-1$.

Before this paper, Conjecture 1.1 was known for quadratic forms of dimension at most 9 [17, Lemma 3.3]. A natural companion to Conjecture 1.1 is the quadratic Zariski problem:

Conjecture 1.2 (Quadratic Zariski problem). - If two quadrics of the same dimension over a field are stably birational, then they are birational.

Conjecture 1.2 is known for quadratic forms of dimension at most 7 , as we will discuss. A general reference on Conjecture 1.2 is [5, Theorem 6.1].

Here are the known results about birational geometry of low-dimensional quadrics in more detail; throughout we consider only anisotropic quadrics over a field. Two anisotropic conics are birational if and only they are isomorphic. Likewise, two anisotropic quadric surfaces are birational if and only if they are isomorphic, by Wadsworth [15, Theorem XII.2.2]. This hides a special phenomenon, however: a Pfister quadric surface is birational to $\mathbf{P}^{1}$ times any conic contained in it, by Knebusch [11, pp. 73-74]. (For example: the quadric surface $x_{0}^{2}-a x_{1}^{2}-b x_{2}^{2}+a b x_{3}^{2}=0$ is birational to $\mathbf{P}^{1}$ times the conic $x_{0}^{2}-$ $a x_{1}^{2}-b x_{2}^{2}=0$, for any $a, b$ in $k^{*}$.) A non-Pfister quadric surface $X$ (there are many equivalent conditions: $X$ has first Witt index 1, or Picard number 1 , or nontrivial discriminant) is not stably birational to any curve. For the definitions of Pfister forms and Pfister neighbors, see section 2.

We state Hoffmann's bound on the first Witt index; several proofs are given in the book by Elman-Karpenko-Merkurjev [3, Theorem 22.5, Corollary 26.6]. The bound is optimal, because equality holds for Pfister neighbors.

Lemma 1.3. - An anisotropic quadratic form of dimension $2^{r}+s$, for $1 \leq$ $s \leq 2^{r}$, has first Witt index at most $s$.

All 5-dimensional anisotropic forms (corresponding to projective quadrics of dimension 3) have first Witt index 1 by Lemma 1.3, and so they are not stably birational to any lower-dimensional variety. Two non-isomorphic 5-dimensional anisotropic forms are stably birational if and only if they are both neighbors of the same Pfister 3-form, by Hoffmann [4, main theorem]. In that case, the two quadrics are in fact birational, by Ahmad-Ohm [1, Corollary 1.6, Theorem 2.5]. Lam's textbook explains Ahmad-Ohm's birational map between the quadrics $\langle 1, a, b, a b, c\rangle$ and $\langle 1, a, c, a c, b\rangle$ [15, Theorem XII.2.15]. The map was generalized by Roussey [16, Proposition 7.2.1], and generalized further in this paper (Lemma 5.1). 
The same thing happens in dimension 6: two 6-dimensional anisotropic forms which are not isomorphic are stably birational if and only if both are neighbors of the same 3-fold Pfister form. The proof for 6-dimensional forms with trivial discriminant (Albert forms) uses Merkurjev's index reduction formula [3, Theorem 30.5], [15, Theorem III.4.8, Corollary XII.2.13]. The proof for nontrivial discriminant is by Laghribi [14, Theorem 1.4(2)]. Two 6-dimensional forms which are neighbors of the same 3-fold Pfister form are birational, again by Ahmad-Ohm [1]. Also, a Pfister neighbor of dimension 6 is birational to $\mathbf{P}^{1}$ times (the quadric associated to) any 5-dimensional subform [1].

In dimension 7, Karpenko (following Izhboldin) determined when two nonsimilar forms $\alpha$ and $\beta$ are stably birational [9, Theorem 3.1, Corollary 3.4]. This happens exactly when the 8-dimensional form $\alpha \perp\left\langle\operatorname{det}_{ \pm}(\alpha)\right\rangle$ is $\operatorname{divisible}$ by a binary form and is similar to $\beta \perp\left\langle\operatorname{det}_{ \pm}(\beta)\right\rangle$. When that happens, Roussey proved that $\alpha$ and $\beta$ are birational [16, Theorem 7.3.1]. Theorems 3.1 and 6.3 below generalize his result.

For quadratic forms of dimension 8 or more, results on birational (as opposed to stably birational) equivalence are much less complete. In particular, two 8dimensional forms $\sigma$ and $\tau$ which are "half-neighbors" ( $a \sigma \perp b \tau$ is a Pfister form for some nonzero $a$ and $b$ ) are stably birational [5, Corollary 2.6]. But it has not been known whether such forms (if non-similar) are birational, even in a single example. We give the first known construction of birational maps between non-isomorphic half-neighbors in section 5 .

All the quadrics in the previously known constructions of birational maps between quadrics, by Ahmad-Ohm and Roussey, are "neighbors" of multiples of Pfister forms, as we have seen in low dimensions. We define these neighbors in the next section.

\section{Neighbors}

In this section we define neighbors of multiples of Pfister forms and the previously known results on their birational classification.

The most important quadratic forms over any field are the Pfister forms, whose definition we now recall. First, for a nonzero element $a$ of a field $k$, the 1-fold Pfister form $\langle\langle a\rangle\rangle$ means the 2-dimensional quadratic form $\langle 1,-a\rangle$, that is, $x_{1}^{2}-a x_{2}^{2}$. For nonzero elements $a_{1}, \ldots, a_{n}$ of $k$, the $n$-fold Pfister form $\left\langle\left\langle a_{1}, \ldots, a_{n}\right\rangle\right\rangle$ means the tensor product $\left\langle\left\langle a_{1}\right\rangle\right\rangle \otimes \cdots \otimes\left\langle\left\langle a_{n}\right\rangle\right\rangle$, a quadratic form of dimension $2^{n}$.

A neighbor of a Pfister form $\rho$ means a form $\varphi$ which is similar to a subform of $\rho$ of dimension greater than half the dimension of $\rho$. We can think of Pfister neighbors as a class of the most special quadratic forms. An anisotropic quadratic form is a Pfister neighbor if and only if it is stably birational to a 
Pfister form (meaning that the corresponding projective quadrics are stably birational), by Hoffmann [3, Theorem 22.5, Corollary 26.6]. Moreover, a Pfister neighbor $\varphi$ (as a quadratic form) uniquely determines the corresponding Pfister form $\rho$ [3, Remark 23.12]. On the other hand, it is not known whether two Pfister neighbors which are stably birational and have the same dimension must be birational. Until this paper, only the following case was known. By definition, a special Pfister neighbor $\varphi$ is a quadratic form that is similar to a form $P_{0} \perp-a P_{1}$ where $P_{0}$ is a Pfister form, $P_{1}$ is a nonzero subform of $P_{0}$, and $a$ is a nonzero element of $k$. Clearly $\varphi$ is a neighbor of the Pfister form $P_{0} \otimes\langle\langle a\rangle\rangle$. The point of the notion is that, by Ahmad-Ohm, any two special Pfister neighbors which are stably birational (equivalently, which are neighbors of the same Pfister form) and which have the same dimension must be birational [1, Theorem 1.6]. Their maps are a special case of the transposition maps defined in Lemma 5.1.

Ahmad-Ohm also showed that every neighbor of codimension at most $n$ of an $n$-fold Pfister form is special, whereas (over suitable fields) neighbors of codimension at least 5 in a 4 -fold Pfister form need not be special [1, 2.5, 2.8]. As a result, all quadratic forms which are Pfister neighbors of dimension at most 8 are special (this explains several of the low-dimensional results in section 1), whereas those of dimension 9 or 10 or 11 need not be special. Thus Pfister neighbors of dimension 9 or 10 or 11 were basic cases where the quadratic Zariski problem remained open. But now Theorem 3.1 solves the ruledness and quadratic Zariski problems, Conjectures 1.1 and 1.2, for all neighbors of an $r$-fold Pfister form when $r$ is at most 4 .

Now we consider a more general class of "neighbors" studied by Ahmad-Ohm and Roussey. First, define a multiple of an $r$-fold Pfister form $\rho$ with $r$ at least 1 to be the tensor product of $\rho$ with some quadratic form; thus we can write a multiple of $\rho$ as $\left\langle b_{1}, \ldots, b_{n}\right\rangle \otimes \rho=b_{1} \rho \perp \cdots \perp b_{n} \rho$ for some nonzero elements $b_{i}$ of the field $k$. Define a neighbor $\sigma$ of a multiple of a Pfister form $\rho$ to be a nondegenerate form which is similar to a subform of some multiple $\varphi \otimes \rho$ of $\rho$ such that $\varphi$ has dimension at least 2 and $\operatorname{dim}(\varphi \otimes \rho)-\operatorname{dim}(\sigma)<\operatorname{dim}(\rho)$. The point of this notion is that a neighbor $\sigma$ of a multiple $\varphi \otimes \rho$ of a Pfister form $\rho$ is stably birational to $\varphi \otimes \rho$, since the first Witt index of $\varphi \otimes \rho$ (if it is anisotropic) is a multiple of the dimension of $\rho$. (If $\varphi \otimes \rho$ is isotropic, then again $\sigma$ is stably birational to $\varphi \otimes \rho$, since both quadrics are rational: either $\rho$ is hyperbolic, in which case $\varphi \otimes \rho$ is hyperbolic and so $\sigma$ is isotropic, or $\rho$ is anisotropic, in which case the Witt index of $\varphi \otimes \rho$ is a multiple of the dimension of $\rho$, and hence $\sigma$ is isotropic.) The standard proof of these results uses function fields [3, Corollary 23.7]. There is also an elementary proof by Wadsworth-Shapiro [19, Theorem $2(\mathrm{c})]$.

TOME $137-2009-\mathrm{N}^{\circ} 2$ 
As in the case of Pfister neighbors, we can ask whether two neighbors of the same multiple of a Pfister form which have the same dimension must be birational. The previously known positive results were restricted to a special case. Define a special neighbor of a multiple of a Pfister form $\rho$ to be a neighbor of the product of $\rho$ with some $d$-dimensional form that contains the product of $\rho$ with some $(d-1)$-dimensional form. (Notice that $\rho$ is part of the data in this definition. We are calling a "special neighbor" what Roussey called a "very special neighbor", for brevity [16, Definition 7.1.3].) Roussey's main result is the following. He used this to complete the solution of the quadratic Zariski problem for all quadratic forms of dimension 7 , as discussed in section 1 .

TheOREM 2.1 (Roussey [16, Theorem 7.3.1]). - Let $q_{1}$ and $q_{2}$ be special neighbors of the same multiple of an $r$-fold Pfister form $\rho$ with $r \leq 3$. If $q_{1}$ and $q_{2}$ have the same dimension, then they are birational.

We have two generalizations of Theorem 2.1. First, we can remove the restriction to $r$ at most 3 (Theorem 6.3). Also, for $r$ at most 3, Theorem 3.1 proves the same result on birational classification for arbitrary neighbors of a multiple of a Pfister form, not just special neighbors.

\section{Construction of birational maps between neighbors}

We now solve the problem of birational classification for all neighbors of an $r$-fold Pfister form with $r$ at most 4 . We also determine exactly which neighbors of an $r$-fold Pfister form with $r$ at most 4 are ruled, thus proving the ruledness conjecture (Conjecture 1.1) in this case. Only the case of special Pfister neighbors had been known before, by Ahmad-Ohm in 1995 [1]. Also, the main result works for arbitrary neighbors of multiples of an $r$-fold Pfister form with $r$ at most 3. (Note that any neighbor of a 4-fold Pfister form can be viewed as a neighbor of a multiple of a 3-fold Pfister form.)

THEOREM 3.1. - Any two neighbors of a given multiple of a r-fold Pfister form with $r$ at most 3 which have the same dimension are birational.

Moreover, let $q$ be any neighbor of a multiple $\varphi \otimes \rho$ of an $r$-fold Pfister form $\rho$ with $r$ at most 3. Then $q$ is birational to the product of projective space of dimension $\operatorname{dim}(q)-\operatorname{dim}(\varphi \otimes \rho)+\operatorname{dim}(\rho)$ with a neighbor of $\varphi \otimes \rho$. In particular, $q$ is ruled if it has codimension at most $\operatorname{dim}(\rho)-2$ in $\varphi \otimes \rho$.

Corollary 3.2. - The ruledness and quadratic Zariski problems, Conjectures 1.1 and 1.2, have a positive solution for neighbors of an r-fold Pfister form with $r$ at most 4. More precisely, if $\sigma$ is a neighbor of an $r$-fold Pfister form with $r$ at most 4 , and $\tau$ is any form of the same dimension which is stably birational to $\sigma$, then $\tau$ is birational to $\sigma$. Moreover, if a quadratic form $\sigma$ is a 
Pfister neighbor of dimension $2^{r}+s, 1 \leq s \leq 2^{r}, r \leq 3$, then $\sigma$ is birational to the product of projective space of dimension $s-1$ with a subquadric of $\sigma$; moreover $s$ here is equal to the first Witt index $i_{1}(\sigma)$.

Proof of Corollary 3.2. - If $\sigma$ is a neighbor of a Pfister form $\rho$ and $\tau$ is any form stably birational to $\sigma$, then $\tau$ is also a neighbor of $\rho$, by Hoffmann [3, Theorem 22.5, Corollary 26.6]. By Theorem 3.1, if $\sigma$ has the same dimension as $\tau$, then they are birational. (Notice that, to apply the theorem, we view $\rho=\left\langle\left\langle a_{1}, \ldots, a_{r}\right\rangle\right\rangle$ for $r \geq 1$ as a multiple of the Pfister form $\left\langle\left\langle a_{1}, \ldots, a_{r-1}\right\rangle\right\rangle$, since the definition of a "neighbor of a multiple $\varphi \otimes \rho$ " requires $\varphi$ to have dimension at least 2.) The ruledness statement is immediate from Theorem 3.1. Finally, for an anisotropic Pfister neighbor $\sigma$ of dimension $2^{r}+s, 1 \leq s \leq 2^{r}$, it is immediate from Hoffmann's bound on the first Witt index, Lemma 1.3, that $i_{1}(\sigma)$ is equal to $s$.

A special case of Corollary 3.2 is that every Pfister neighbor of codimension at most 7 satisfies the ruledness and quadratic Zariski problems, Conjectures 1.1 and 1.2. Indeed, these conjectures hold for all neighbors of an $r$-fold Pfister form with $r \leq 3$. A neighbor of codimension at most 7 of an $r$-fold Pfister form with $r \geq 4$, on the other hand, can be viewed as a neighbor of a multiple of a 3 -fold Pfister form, and so again Corollary 3.2 proves the results we want. Thus these conjectures hold for all Pfister neighbors of dimension at most 16 , as well as for those of dimensions from 25 to 32 , and so on. In very high dimensions, however, Corollary 3.2 gives less about Pfister neighbors than Ahmad-Ohm's result that a neighbor of codimension at most $r$ in an $r$-fold Pfister form is special and therefore satisfies the two conjectures [1, Corollary 2.5].

Proof of Theorem 3.1. - For $k$ finite, every anisotropic quadratic form has dimension at most 2 and the theorem is trivially true. So we can assume that $k$ is infinite.

Write the given multiple of the Pfister form $\rho$ as $\left\langle a_{1}, \ldots, a_{n}\right\rangle \otimes \rho$, where $n \geq 2$. Since $r$ is at most 3, the $r$-fold Pfister form $\rho$ admits a bilinear "multiplication" $x y$ on the vector space of $\rho$ such that $\rho(x y)=\rho(x) \rho(y)$ [15, Theorem X.2.11], and for generic $x$ the map $y \mapsto x y$ is invertible. Ahmad-Ohm introduced a rational map $F$ from the quadric $\left\langle a_{1}, \ldots, a_{n}\right\rangle \otimes \rho$ to the quadric $\left\langle a_{1}, \ldots, a_{n-1}\right\rangle \otimes$ $\rho \perp\left\langle a_{n}\right\rangle$, defined by

$$
\left[x_{1}, \ldots, x_{n-1}, y\right] \mapsto\left[y x_{1}, \ldots, y x_{n-1}, \rho(y)\right] .
$$

[1, after 1.3]. The map $F$ is dominant, and its general fibers are linear spaces. Therefore the first quadric is birational to the product of projective space of $\operatorname{dimension} \operatorname{dim}(\rho)-1$ with the second quadric. (The latter statement is true for $r$-fold Pfister forms for any $r$. The advantage of $r \leq 3$ is that the general 
fibers are linear subspaces in projective space, not just isomorphic to projective spaces.)

Let $q$ be any neighbor of $\varphi \otimes \rho=\left\langle a_{1}, \ldots, a_{n}\right\rangle \otimes \rho$. That is, after scaling, $q$ is a nondegenerate subform of codimension less than $\operatorname{dim}(\rho)$ in $\varphi \otimes \rho$. The projective quadric $Q$ corresponding to $q$ is the intersection of the quadric corresponding to $\varphi \otimes \rho$ with a linear subspace. Suppose that the rational map $F$ is defined at some point of $Q$, and that the derivative of $F$ restricted to $Q$ is surjective at that point. Then the restriction of $F$ to $Q$ is dominant, and its general fibers are still linear subspaces. That will imply that $q$ is birational to the product of projective space of the appropriate dimension with the quadric $\left\langle a_{1}, \ldots, a_{n-1}\right\rangle \otimes$ $\rho \perp\left\langle a_{n}\right\rangle$. Since the latter quadric does not depend on the form $q$, that will complete the proof.

It remains to show that for any nondegenerate subform $q$ of $\varphi \otimes \rho$ with codimension less than $\operatorname{dim}(\rho), q$ can be embedded in $\varphi \otimes \rho$ in such a way that the rational map $F$ is defined at some point of the corresponding quadric $Q$ and that $F$ restricted to $Q$ has surjective derivative at that point.

Consider the Grassmannian of all linear subspaces of $\varphi \otimes \rho$ of dimension equal to $\operatorname{dim}(q)$. An open dense subset of this Grassmannian corresponds to subspaces whose intersection with the quadric $\varphi \otimes \rho=0$ is smooth, meets the open set where $F$ is defined, and such that $F$ restricted to this intersection has surjective derivative at some point. (Here we use the assumption that $\operatorname{dim}(q)$ is at least the dimension of the form $\left\langle a_{1}, \ldots, a_{n-1}\right\rangle \otimes \rho \perp\left\langle a_{n}\right\rangle$.) Then the desired conclusion follows from Lemma 3.3.

LEMMA 3.3. - Let $\alpha$ be any nondegenerate subform of a nondegenerate quadratic form $\beta$ over an infinite field $k$. Let $U$ be a nonempty open subset of the Grassmannian of linear subspaces of the vector space $\beta$ of dimension $\operatorname{dim}(\alpha)$. Then there is an isometry of $\beta$ which moves $\alpha$ to a subspace that corresponds to a point of $U$.

Proof. - By Witt's cancellation theorem, the orthogonal group $O(\beta)$ over the algebraic closure of $k$ acts transitively on the open dense subset of the Grassmannian corresponding to subspaces whose intersection with the quadric is smooth (equivalently, subspaces on which the quadratic form is nondegenerate). The group of $k$-points $O(\beta)(k)$ is Zariski dense in the whole orthogonal group, using that $k$ is infinite and the special orthogonal group is connected [2, Corollary 18.3]. Therefore, there is an element of $O(\beta)(k)$ which moves the given subspace $\alpha \subset \beta$ into the given open set $U$. 


\section{The ruledness conjecture and the structure of 14-dimensional forms}

If a quadric is ruled, then Karpenko showed that its first Witt index is greater than 1, and we conjecture the converse. More precisely, Conjecture 1.1 predicts that any quadric $X$ is birational to the product of some subquadric with projective space of dimension $i_{1}(X)-1$. Using the results of this paper, we prove this conjecture in a larger range of dimensions than before, as follows.

Theorem 4.1. - The ruledness conjecture, Conjecture 1.1, holds for odddimensional quadratic forms of dimension at most 17, and also for evendimensional forms of dimension at most 10 or equal to 14.

Proof. - Throughout the proof, we consider only anisotropic forms. In dimensions at most 9 , the ruledness conjecture was known [17].

There is an appealing dichotomy for 10-dimensional forms: if $i_{1}(q)>1$, then $q$ is either a Pfister neighbor or divisible by a binary form, by Izhboldin [7, proof of Conjecture 0.10]. We know that $i_{1}(q)=2$ by Hoffmann's bound for $i_{1}$, Lemma 1.3. If $q$ is divisible by a binary form, then $q$ is ruled by AhmadOhm (see Lemma 6.1). If $q$ is a Pfister neighbor, then Corollary 3.2 shows the new result that $q$ is ruled, even though $q$ need not be a special Pfister neighbor.

The ruledness conjecture for 12-dimensional forms $q$ remains open. In what follows we use Karpenko's determination of all possible values of the first Witt index for anisotropic forms of a given dimension, although the cases we need were known earlier [3, Theorem 79.9]. For a 12-dimensional form, if the first Witt index is greater than 1 , then it is either 2 or 4 . If $i_{1}(q)=4$, then $q$ is a Pfister neighbor by Kahn [8, Theorem 4], simplified by Izhboldin [6, Corollary 1.6]. So Conjecture 1.1 on ruledness holds by Corollary 3.2. That is, the corresponding projective quadric is birational to $\mathbf{P}^{3}$ times a 7 -dimensional quadric. If $i_{1}(q)=2$, then the ruledness conjecture would follow from Vishik's conjecture that such a form $q$ must be divisible by a binary form [18, p. 95].

We now prove Conjecture 1.1 on ruledness for forms of odd dimension at most 17. For forms $q$ of dimension 11 or 13, having first Witt index greater than 1 implies that $q$ is a Pfister neighbor by Kahn [8, Theorem 4], simplified by Izhboldin [6, Corollary 1.6]. Thus we know the ruledness conjecture by Corollary 3.2. Next, a 15-dimensional form with $i_{1}>1$ has $i_{1}=7$ or $i_{1}=3$. If $i_{1}=7$, then $q$ is a Pfister neighbor by Knebusch and the ruledness conjecture holds. If $i_{1}=3$, then Vishik showed that $q \perp\left\langle\operatorname{det}_{ \pm}(q)\right\rangle$ is a multiple of a 2 -fold Pfister form [18, p. 79]. Therefore $q$ is a neighbor of the type covered by Theorem 3.1, and so the ruledness conjecture holds. Finally, for $q$ of dimension 17, we have $i_{1}=1$ by Hoffmann's bound, Lemma 1.3, and so the ruledness conjecture is vacuously true.

TOME $137-2009-\mathrm{N}^{\mathrm{O}} 2$ 
Finally, Conjecture 1.1 on ruledness for dimension 14 follows from Theorem 4.2 below, in view of Theorem 3.1.

The following result is a satisfying extension of Izhboldin's theorem on 10dimensional forms to dimension 14. The proof uses Vishik's results and methods, but they have to be combined in an elaborate way.

THEOREM 4.2. - Let $q$ be a 14-dimensional anisotropic quadratic form with first Witt index greater than 1. Then $q$ is either

(1) a Pfister neighbor, and $i_{1}(q)=6$,

(2) divisible by a binary form, and $i_{1}(q)=2$, or

(3) a subform of a 16-dimensional multiple of a 2-fold Pfister form, and $i_{1}(q)=2$.

Vishik has shown that Theorem 4.2 does not extend to dimension 16: there is a 16-dimensional form with first Witt index 2 which is not divisible by a binary form and hence, for dimension reasons, is not a neighbor of any multiple of a Pfister form (Theorem 7.1). Nonetheless, we will prove the ruledness conjecture for Vishik's example in Theorem 7.2.

Although cases (2) and (3) in Theorem 4.2 overlap, neither can be omitted. In particular, over a rational function field $k=k_{0}(a, b, c, d, e, f)$, the 14-dimensional form $\langle\langle a, b\rangle\rangle \otimes\langle c, d, e, f\rangle-\langle c, d\rangle$ satisfies (3). But it is not divisible by a binary form, because it has Witt index 3 over the extension field $k(\sqrt{-c d})$, with anisotropic part the 8-dimensional form $\langle\langle a, b\rangle\rangle \otimes\langle e, f\rangle$. (A multiple of a binary form is either hyperbolic or has even Witt index, by the results mentioned in section 2.)

Proof of Theorem 4.2. - For an anisotropic form of dimension 14, Karpenko's theorem gives that the first Witt index is 1,2 , or 6 [3, Theorem 79.9]. If $i_{1}(q)=6$, then $q$ is a Pfister neighbor by Knebusch [12, Corollary 8.2] and we are done. So we can assume that $i_{1}(q)=2$.

Next, we will show that $q$ must have splitting pattern either $(2,2,2,1)$ or $(2,1,1,2,1)$. We use Chow groups as in [3] to prove this, although the argument could also be formulated in terms of motivic decompositions as in [18]; the equivalence between the two languages is described in [3, Theorem 94.1]. Let $Q$ be the projective quadric of dimension 12 associated to $q$. For any projective quadric, write $D=\operatorname{dim} Q$ (in the current example, $D=12$ ) and $d=\lfloor D / 2\rfloor$. A basis for the Chow groups modulo 2 of $Q^{2}$ over the algebraic closure $\bar{k}$ is given by the products of powers $h^{i}$ and classes $l_{i}$ for $0 \leq i \leq d$, where $h$ denotes the hyperplane class and $l_{i}$ denotes a linear space of dimension $i$ on $X_{\bar{k}}$. A basic discrete invariant of a quadratic form is the image of the group of algebraic cycles on $Q^{2}$ (defined over $k$ ) in the Chow groups modulo 2 of $Q_{\bar{k}}^{2}$, viewed as 
a vector space over $\mathbf{F}_{2}$. Here the cycles $h^{i} \times h^{j}$ are always defined over $k$, and so we simply remove them from any cycle we consider. The remaining basis elements of the middle dimension and above for $Q_{\bar{k}}^{2}$ are pictured below.

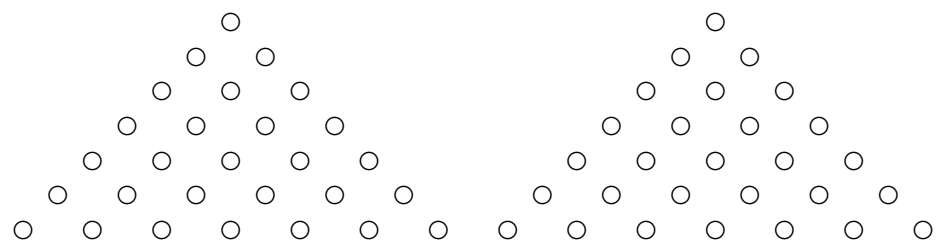

The rows represent the cycles of a given dimension, with the bottom representing middle-dimensional cycles. The $i$ th row from the bottom starts with the classes $h^{0} \times l_{i}, \ldots, h^{d-i} \times l_{d}$, and then come the classes $l_{d} \times h^{d-i}, \ldots, l_{i} \times h^{0}$. For $D$ even, the middle-dimensional basis element $l_{d} \times l_{d}$ does not occur in the decomposition of any cycle defined over $k$ for $Q$ not hyperbolic [3, Lemma 73.2], and so is not pictured.

By [3, Lemma 73.13], all cycles of the middle dimension or above on $Q^{2}$, viewed over $\bar{k}$, can only involve basis elements which lie in a sequence of triangles determined by the splitting pattern. For example, if $q$ had splitting pattern $(2,1,3,1)$, these "shell triangles" would be:

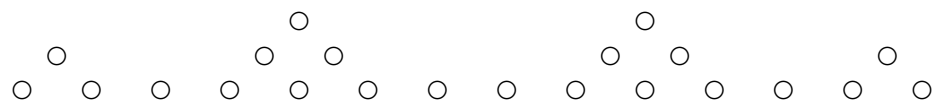

By [3, Lemma 73.20], since $i_{1}(Q)=2$, there is a 13-dimensional cycle $\alpha$ on $Q^{2}$ whose class over $\bar{k}$ contains $h^{0} \times l_{1}$, the top of the first shell triangle. By [3, Corollary 80.14] (on "outer excellent connections"), it follows that $\alpha$ also contains $l_{5} \times h^{4}$, shown as a black dot in the picture.

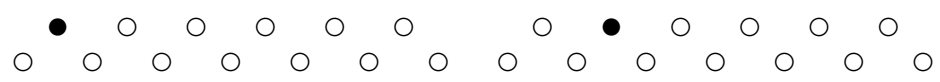

(This theorem is the deepest ingredient of the proof, in that it uses the Steenrod operations on Chow groups modulo 2.) By the above statement on shell triangles, it follows that $i_{h}(q)$ is not 2 and that if $i_{h}(q)$ is 1 , then $i_{h-1}(q)$ is at least 2 . Here $h$ denotes the height of $q$, meaning the length of the splitting pattern $\left(i_{1}, \ldots, i_{h}\right)$.

We will use systematically what is known about low-dimensional forms. In particular, the possible splitting patterns in dimension 10 are $(2,2,1),(2,1,2)$, $(2,1,1,1),(1,2,2),(1,1,2,1),(1,1,1,2)$, and $(1,1,1,1,1)$ [18]. The splitting pattern of $q$ must be 2 followed by one of these sequences, and the previous paragraph leaves only two possibilities, $(2,2,2,1)$ or $(2,1,1,2,1)$.

TOME $137-2009-\mathrm{N}^{\mathrm{O}} 2$ 
An anisotropic form $q$ of dimension congruent to 2 modulo 4 is divisible by a binary form if and only if it has splitting pattern (even, ..., even, 1), by Knebusch's results [18, Theorem 7.1, Theorem 7.5]. More precisely, in that case, $q$ is divisible by $\left\langle\left\langle\operatorname{det}_{ \pm} q\right\rangle\right\rangle$. Thus, if our form $q$ has splitting pattern $(2,2,2,1)$, then it is divisible by a binary form, as we want.

It remains to show that if $q$ has splitting pattern $(2,1,1,2,1)$, then $q$ is a codimension-2 subform of a multiple of a 2-fold Pfister form, $\langle\langle a, b\rangle\rangle \otimes\langle c, d, e, f\rangle$. To begin, we claim that over $K:=k\left(\sqrt{\operatorname{det}_{ \pm} q}\right), q$ has trivial Clifford invariant in $H^{2}\left(K, \mathbf{F}_{2}\right)[3$, section 14] as well as trivial discriminant. (Merkurjev's theorem says that these properties are equivalent to $q_{K}$ being in the ideal $I^{3}$ of the Witt ring [3, section 16], but we do not need that here.) To see that $q_{K}$ has trivial Clifford invariant, we use Knebusch's theorem that the splitting pattern of $q$ over any field extension is a "specialization" of that of $q$, meaning that it is obtained by possibly adding some sets of adjacent entries and possibly removing some initial entries [18, Theorem 7.5]. Over the quadratic extension $K$, the last entry of the splitting pattern of $q_{K}$ must be a power of 2 at least 2, but we see that it cannot be 2 , and so it must be at least 4 . This means that $q_{K}$ has trivial discriminant and Clifford invariant [3, Proposition 25.14].

It follows that the Clifford invariant of $q$ in $H^{2}\left(k, \mathbf{F}_{2}\right)$ is killed by passage to $k\left(\sqrt{\operatorname{det}_{ \pm} q}\right)$, and so we can write $c(q)=\left\{\operatorname{det}_{ \pm} q, b\right\}$ for some $b$ in $k^{*}[3$, Theorem 99.13]. As a result, the 16-dimensional form $\varphi=q \perp\left\langle b,-b \operatorname{det}_{ \pm} q\right\rangle$ has trivial discriminant and Clifford invariant.

Now let $p$ be a 15-dimensional form that contains $q$ and is contained in $\varphi$. Since $\varphi$ has trivial discriminant and Clifford invariant, the highest Witt index $i_{h}(p)$ must be 3 , and so the splitting pattern of $p$ is either $(3,1,3),(1,1,1,1,3)$ or (if $p$ is isotropic) $(1,1,1,3)$, by Vishik [18, Table 1, p. 84]. We will show that it must be $(3,1,3)$. Note that this does not follow just from $p$ being of codimension 1 in a form with trivial discriminant and Clifford invariant; we need to use what we know about the 14-dimensional form $q$ that $p$ contains.

As a preliminary, we continue our analysis of the Chow groups of $Q^{2}$, using that $q$ has splitting pattern $(2,1,1,2,1)$; this could all be said equivalently in terms of motivic decompositions of $Q$. We have already shown, using $i_{1}(Q)=2$, that there is a 7-dimensional cycle $\alpha$ on $Q^{2}$ whose class over $\bar{k}$ contains $h^{0} \times l_{1}$, the top of the first shell triangle, as well as $l_{5} \times h^{4}$, the top of the fourth shell triangle from the right. By [3, Corollary 73.23], the components of any cycle $y$ on $Q^{2}$ occur in pairs: if a basis element at position $i$ from the left in the $j$ th shell triangle occurs in $y$, then so does the basis element at position $i$ from the left in the $j$ th shell triangle from the right. So $\alpha$ contains the four basis elements pictured. 
In view of the lemma on shell triangles, the class of $\alpha$ cannot involve any other basis elements, and so it is the sum of the four basis elements shown, $h^{0} \times l_{1}, h^{4} \times l_{5}, l_{5} \times h^{4}$, and $l_{1} \times h^{0}$.

Now suppose that the 15-dimensional form $p$ containing $q$ has splitting pattern $(1,1,1,1,3)$ or $(1,1,1,3)$. Let $P$ be the quadric associated to $p$. Because $q$ has first Witt index 2 , we see that over every extension field of $k$, the conditions $i_{W}(p)>1$ and $i_{W}(q)>0$ on the Witt indices are equivalent. Therefore, by [18, Theorem 4.15], there is a middle-dimensional cycle $\beta$ on $P^{2}$ associated to $\alpha$, consisting (in $C H_{*}\left(P_{\bar{k}}^{2}\right) / 2$ ) of the sum of the basis elements $h^{1} \times l_{1}, h^{5} \times l_{5}$, $l_{5} \times h^{5}$, and $l_{1} \times h^{1}$.

Recall the definition of the splitting tower $k_{0}, \ldots, k_{h}$ of $p$ from section 1 . Let $E$ be the field $k_{2}$ if $p$ has splitting pattern $(1,1,1,1,3)$ or $k_{1}$ if $p$ is isotropic with splitting pattern $(1,1,1,3)$. Then $r:=\left(p_{E}\right)_{\text {an }}$ has dimension 11 and splitting pattern $(1,1,3)$; let $R$ be the corresponding projective quadric. The cycle $\beta$ on $P_{E}$ determines a middle-dimensional cycle $\gamma$ on $R$ which consists (in $C H_{*}\left(R_{\bar{E}}^{2}\right) / 2$ ) of the sum of the basis elements $h^{3} \times l_{3}$ and $l_{3} \times h^{3}[3$, Remark $72.5]$.

(This comes from a correspondence which chops off the left two and right two dots, in the decomposition of a given cycle on $P^{2}$.) But this contradicts what is known about the discrete invariants of an 11-dimensional form $r$ with splitting pattern $(1,1,3)$. Namely, Vishik showed that the class of every middle-dimensional cycle on $R^{2}$ is a linear combination of the two sums of basis elements shown [18, Lemma 7.10].

Again, one can refer to [3, Theorem 94.1] and [18, Proposition 3.5] for the translation between Chow groups and motives.

This contradiction shows that the 15-dimensional form $p$ containing our form $q$ must have splitting pattern $(3,1,3)$. But Vishik showed that for a 15-dimensional form $p$ with splitting pattern $(3,1,3)$, the 16-dimensional form $p \perp\left\langle\operatorname{det}_{ \pm} p\right\rangle$ is a multiple of a 2 -fold Pfister form, $\langle\langle a, b\rangle\rangle \otimes\langle c, d, e, f\rangle[18$, p. 81]. Thus the 14-dimensional form $q$ is a codimension-2 subform of a multiple of a 2-fold Pfister form. 


\section{Transposition maps}

In this section, we construct a new family of birational maps between two quadrics. For any Pfister form $\rho$ over a field $k$, any form $\varphi$, any two subforms $\sigma$ and $\tau$ of $\rho$ on which the quadratic form is not zero, and any $b, c$ in $k^{*}$, we define a birational equivalence

$$
\varphi \otimes \rho \perp b \sigma \perp c \tau \underset{\text { bir }}{\sim} \varphi \otimes \rho \perp c \sigma \perp b \tau .
$$

We call these maps "transpositions." They generalize all the previously known birational maps between different quadrics: Ahmad-Ohm's map between two special Pfister neighbors, and Roussey's map between two special neighbors of a multiple of a Pfister form, which can be viewed as the case where the subform $\sigma$ is all of $\rho$. The maps here do not include the other maps introduced in Corollary 3.2, birational maps between arbitrary neighbors rather than just special neighbors.

The new transposition map is not restricted to neighbors of multiples of Pfister forms. In particular, it gives the first birational equivalence between two half-neighbors which are not similar. By definition, two forms $\varphi$ and $\psi$ of dimension $2^{n}$ are called half-neighbors (of each other) if $d \varphi \perp e \psi$ is a Pfister form for some $d, e$ in $k^{*}$. It is elementary that two half-neighbors are stably birational [5, Corollary 2.6], and this is a prominent case where stable birational equivalence is not known to imply birational equivalence. In particular, Hoffmann showed that over a rational function field $k=k_{0}\left(a, b, x_{1}, x_{2}\right)$ or $k=k_{0}((a))((b))\left(\left(x_{1}\right)\right)\left(\left(x_{2}\right)\right)$, the following 8-dimensional forms are halfneighbors but not similar. Take $\pi=\left\langle\left\langle-a,-x_{1},-x_{2}\right\rangle\right\rangle, \tau=\left\langle-1,-a, b x_{1}, a b x_{2}\right\rangle$, $\varphi=(\tau \perp \pi)_{\text {an }}$, and $\psi=(\tau \perp-b \pi)_{\text {an }}$. That is,

$$
\begin{aligned}
\varphi & =\left\langle x_{1}, x_{2}, a x_{1}, a x_{2}, x_{1} x_{2}, a x_{1} x_{2}, b x_{1}, a b x_{2}\right\rangle \\
& =x_{1}\left\langle\left\langle-a,-x_{1} x_{2}\right\rangle\right\rangle \perp x_{1} x_{2}\langle\langle-a\rangle\rangle \perp b x_{1}\left\langle\left\langle-a x_{1} x_{2}\right\rangle\right\rangle
\end{aligned}
$$

and

$$
\begin{aligned}
-b x_{1} \psi & =\left\langle x_{1}, x_{2}, a x_{1}, a x_{2}, b x_{1}, a b x_{1}, x_{1} x_{2}, a\right\rangle \\
& =x_{1}\left\langle\left\langle-a,-x_{1} x_{2}\right\rangle\right\rangle \perp b x_{1}\langle\langle-a\rangle\rangle \perp x_{1} x_{2}\left\langle\left\langle-a x_{1} x_{2}\right\rangle\right\rangle .
\end{aligned}
$$

So the new transposition map shows that $\varphi$ and $\psi$ are birational. It remains an open question whether all pairs of two 8-dimensional half-neighbors are birational, however. Dimension 8 is the lowest where the quadratic Zariski problem, Conjecture 1.2, remains open.

Lemma 5.1. - For any Pfister form $\rho$ over a field $k$, any form $\varphi$, any two subforms $\sigma$ and $\tau$ of $\rho$ on which the quadratic form is not zero, and any $b, c$ in 
$k^{*}$, there is a birational equivalence

$$
\varphi \otimes \rho \perp b \sigma \perp c \tau \underset{b i r}{\sim} \varphi \otimes \rho \perp c \sigma \perp b \tau .
$$

Proof. - A Pfister form $\rho$ is strongly multiplicative: there is a "multiplication" $x y$ on the vector space of $\rho$ which is a rational function of $x$ and (for generic $x$ ) invertible linear in $y$ such that $\rho(x y)=\rho(x) \rho(y)$ [15, Theorem X.2.11]. We can also assume that $(a x)(y)=a(x y)$ for $a \in k$ (use the given product for $x$ in an affine hyperplane, and scale the product elsewhere to make this property hold).

Write $\varphi=\left\langle a_{1}, \ldots, a_{n}\right\rangle, n \geq 0$. In terms of a multiplication as above, we define the birational map by

$$
\left[r_{1}, \ldots, r_{n}, s, t\right] \mapsto\left[t\left(s r_{1}\right), \ldots, t\left(s r_{n}\right), \rho(t) s, \rho(s) t\right] .
$$

Here $r_{1}, \ldots, r_{n}$ belong to the vector space $\rho$, while $s$ and $t$ are in the linear subspaces $\sigma$ and $\tau$, respectively.

To show that this map is birational, we would like to define the inverse by

$$
\left[u_{1}, \ldots, u_{n}, v, w\right] \mapsto\left[v^{-1}\left(w^{-1} u_{1}\right), \ldots, v^{-1}\left(w^{-1} u_{n}\right), \rho(v)^{-1} v, \rho(w)^{-1} w\right] .
$$

Note that $v^{-1}$ in the formula does not mean an element of the vector space $\rho$, but merely the inverse of the linear map $x \mapsto v x$. Here $\rho(v)$ and $\rho(w)$ are nonzero for generic $v$ and $w$ in the subspaces $\sigma$ and $\tau$ by assumption. But for this formula to make sense, we also need to know that some point $v$ in $\sigma$ lies in the open set of points such that the linear map $x \mapsto v x$ is defined and invertible, and likewise for $\tau$. Since the quadratic form on $\sigma$ (say) is nonzero, it has a subspace (say 1-dimensional) on which the quadratic form is nondegenerate. Applying Lemma 3.3 to that subspace gives a new embedding of the quadratic form $\sigma$ into $\rho$ with the desired property.

\section{Birational maps between special neighbors}

In this section, Theorem 6.3 determines the birational type of special neighbors of multiples of Pfister forms. When the Pfister form is $r$-fold with $r$ at most 3 , Theorem 3.1 is a more general result, covering arbitrary neighbors. One advantage of special neighbors even for $r$ at most 3 is that all the birational maps we need are composites of certain very explicit "transposition maps" between two quadrics defined by Roussey, where one can describe exactly what is being blown up and blown down.

First, we recall Ahmad-Ohm's observation [1, after 1.3] on the ruledness of special neighbors in a multiple of a Pfister form. The proof is modeled on Knebusch's proof for the case of special Pfister neighbors [11, pp. 73-74]. The general proof is written out in [17, Lemma 3.2], for example. 
Lemma 6.1. - Let $\rho$ be a Pfister form, $\varphi$ any quadratic form of dimension at least $1, \eta$ a nonzero subform of $\rho$, a an element of $k^{*}$. Then the quadric associated to $\varphi \otimes \rho \perp$ a $\eta$ is birational to projective space of dimension $\operatorname{dim}(\eta)-1$ times the quadric associated to $\varphi \otimes \rho \perp\langle a\rangle$.

The key birational maps we use to prove Theorem 6.3 are the transposition maps of Lemma 5.1, which were defined in the case at hand by Roussey. He also proved a criterion for when two special neighbors are similar, which we state for the reader's convenience although it is not needed for what follows.

Lemma 6.2 (Transposition lemma). - Let $\rho$ be a Pfister form, $\varphi$ any quadratic form, and $c$ and $d$ nonzero elements of $k$. Then the special neighbors $\varphi \otimes \rho \perp c \rho \perp\langle d\rangle$ and $\varphi \otimes \rho \perp d \rho \perp\langle c\rangle$ are birational.

Moreover, for $\rho$ of dimension at least 2, these two forms are similar if and only if cd belongs to the group of similarity factors of $\varphi \otimes \rho$.

To prove Theorem 2.1, Roussey uses the birational maps of Lemma 6.2 together with another class of maps that are only defined when $\rho$ is an $r$-fold Pfister form with $r \leq 3$. It turns out, however, that just by repeated use of the maps in Lemma 6.2, we can prove the following statement, with no restriction on $r$.

THEOREM 6.3. - Let $q_{1}$ and $q_{2}$ be special neighbors of the same multiple of a Pfister form $\rho$. If $q_{1}$ and $q_{2}$ have the same dimension, then they are birational.

Proof. - By Lemma 6.1, it suffices to consider quadratic forms of the form $\sigma \otimes \rho \perp\langle a\rangle$. That is, if $(\sigma \oplus\langle a\rangle) \otimes \rho$ is isomorphic to $(\tau \oplus\langle b\rangle) \otimes \rho$, with $\sigma$ of dimension at least 1 , then we have to show that $\sigma \otimes \rho \perp\langle a\rangle$ and $\tau \otimes \rho \perp\langle b\rangle$ are birational. For $\sigma$ of dimension 1 (so the forms we consider are special Pfister neighbors), this was proved by Ahmad-Ohm [1, Theorem 1.6]. We remark that all the birational maps they used are composites of the maps provided by the transposition lemma, Lemma 6.2 (which they proved for $\sigma$ of dimension 1). We can therefore assume that $\sigma$ has dimension at least 2 .

Wadsworth and Shapiro proved the following chain lemma, characterizing when two multiples of a Pfister form are isomorphic, by a quick elementary argument [19, Theorem 1].

Lemma 6.4. - Let $\rho$ be a Pfister form over a field $k$. Two multiples $\left\langle a_{1}, \ldots, a_{m}\right\rangle \otimes \rho$ and $\left\langle b_{1}, \ldots, b_{m}\right\rangle \otimes \rho$ of $\rho$ are isomorphic if and only if the sequence $a_{1}, \ldots, a_{m}$ of elements of $k^{*}$ can be transformed into the sequence $b_{1}, \ldots, b_{m}$ by the following moves.

(1) For $1 \leq i<j \leq m$, we can change $a_{i}, a_{j}$ to any other $b_{i}, b_{j}$ in $k^{*}$ such that $\left\langle a_{i}, a_{j}\right\rangle \cong\left\langle b_{i}, b_{j}\right\rangle$.

(2) For $1 \leq i \leq m$, we can change $a_{i}$ to $c a_{i}$ for any $c \in G(\rho)$. 
Let us say that $\left\langle a_{1}, \ldots, a_{m} ; a_{m+1}\right\rangle \sim\left\langle b_{1}, \ldots, b_{m} ; b_{m+1}\right\rangle$, for nonzero elements $a_{i}$ and $b_{i}$ of the field $k$, if the forms $\left\langle a_{1}, \ldots, a_{m}\right\rangle \otimes \rho \perp\left\langle a_{m+1}\right\rangle$ and $\left\langle b_{1}, \ldots, b_{m}\right\rangle \otimes \rho \perp\left\langle b_{m+1}\right\rangle$ are birational. Thus, we need to show that for $m \geq 2$, if $\left\langle a_{1}, \ldots, a_{m+1}\right\rangle \otimes \rho$ is isomorphic to $\left\langle b_{1}, \ldots, b_{m+1}\right\rangle \otimes \rho$, then $\left\langle a_{1}, \ldots, a_{m} ; a_{m+1}\right\rangle \sim\left\langle b_{1}, \ldots, b_{m} ; b_{m+1}\right\rangle$.

By Lemma 6.4, it suffices to prove this birational equivalence when we perform any of the moves (1) or (2) on a sequence $\left(a_{1}, \ldots, a_{m}, a_{m+1}\right)$. If we multiply $a_{i}$ by an element of $G(\rho)$ for $i \leq m$, then we do not change the form $\left\langle a_{1}, \ldots, a_{m}\right\rangle \otimes \rho \perp\left\langle a_{m+1}\right\rangle$, and so we certainly have the desired birational equivalence. If we multiply $a_{m+1}$ by an element of $G(\rho)$, then the form $\left\langle a_{1}, \ldots, a_{m}\right\rangle \otimes \rho \perp\left\langle a_{m+1}\right\rangle$ changes by a scalar, and so again we have a birational equivalence. This covers move (2). Performing move (1) on two of the numbers $a_{1}, \ldots, a_{m}$ does not change the form we are considering. Finally, by permuting $a_{1}, \ldots, a_{m}$ if necessary, it remains to show: if $\left\langle c_{1}, c_{2}\right\rangle$ is isomorphic to $\left\langle d_{1}, d_{2}\right\rangle$, then

$$
\left\langle a_{1}, \ldots, a_{m-1}, c_{1} ; c_{2}\right\rangle \sim\left\langle a_{1}, \ldots, a_{m-1}, d_{1} ; d_{2}\right\rangle .
$$

We prove that as follows, using $m \geq 2$.

$$
\begin{aligned}
\left\langle a_{1}, \ldots, a_{m-1}, c_{1} ; c_{2}\right\rangle & \sim\left\langle a_{1}, \ldots, a_{m-2}, c_{1}, a_{m-1} ; c_{2}\right\rangle \\
& \sim\left\langle a_{1}, \ldots, a_{m-2}, c_{1}, c_{2} ; a_{m-1}\right\rangle \text { by transposition lemma } \\
& \sim\left\langle a_{1}, \ldots, a_{m-2}, d_{1}, d_{2} ; a_{m-1}\right\rangle \\
& \sim\left\langle a_{1}, \ldots, a_{m-2}, d_{1}, a_{m-1} ; d_{2}\right\rangle \text { by transposition lemma } \\
& \sim\left\langle a_{1}, \ldots, a_{m-1}, d_{1} ; d_{2}\right\rangle .
\end{aligned}
$$

That is, the birational map we want is a composite of two of the birational maps between quadrics provided by the transposition lemma, Lemma 6.2. Theorem 6.3 is proved.

\section{Ruledness of Vishik's example}

Vishik recently gave an example of an anisotropic quadratic form $q$ of dimension 16 which has first Witt index greater than 1 but is not divisible by a binary form. (Since the dimension is a power of 2 , it follows that $q$ is not a neighbor of any multiple of a Pfister form.) Thus Conjecture 1.1 on ruledness predicts that $q$ is ruled, but all previous constructions of anisotropic quadrics which are ruled have only applied to neighbors of multiples of Pfister forms, making this a natural test case. In this section, we show that this form is indeed ruled, in fact by lines in the quadric. The formula we use to prove this is related to the transposition map of Lemma 5.1, but is notably more complex. 
One natural context for Vishik's example is the problem of characterizing the quadratic forms which are divisible by binary forms. If $q$ is divisible by a binary form, then the higher Witt indices $i_{1}, \ldots, i_{h}$ are all even except possibly the last, by the results mentioned in section 2. For quadratic forms $q$ of dimension congruent to 2 modulo 4, it follows from Knebusch's results that the converse holds: if $i_{1}, \ldots, i_{h-1}$ are all even (in this case, $i_{h}$ will equal 1 ), then $q$ is divisible by the binary form $\left\langle\left\langle\operatorname{det}_{ \pm} q\right\rangle\right\rangle[18$, Theorem 7.1, Theorem 7.5]. For forms of dimension 4 or 8 , if the higher Witt indices are all even, then again $q$ is divisible by a binary form, and the same is conjectured for $q$ of dimension 12 [18, p. 95]. A naive generalization of that conjecture is destroyed by the following example.

LEMMA 7.1 (Vishik). — The 16-dimensional form

$$
q=\langle\langle-d,-e,-f\rangle\rangle \perp b\langle 1, d, e, f\rangle \perp c\langle 1, d, e, f\rangle
$$

over the rational function field $k=k_{0}(b, c, d, e, f)$ is anisotropic, with splitting pattern $(2,2,2,2)$, but is not divisible by a binary form.

Proof. - We begin by computing the splitting pattern of $q$. If we extend the base field by adjoining the square root of $c(b d e f)$, then $q$ becomes the anisotropic Pfister form $\langle\langle-d,-e,-f,-b\rangle\rangle$, and so $q$ is anisotropic of dimension 16 over $k$. Moreover, we can write down other field extensions over which $q$ has anisotropic part of dimension 12,8 , or 4 : adjoin the square root of $c(-b d)$, the square root of $c(-b)$, or the square roots of $-b$ and $c(-d)$.

Next, we observe that for every field extension $E$ of $k$ such that $q_{E}$ has anisotropic part of dimension less than $16, q_{E}$ becomes divisible by the binary form $\langle\langle c(b d e f)\rangle\rangle$. Indeed, over $E(\sqrt{c(b d e f)}, q$ becomes the Pfister form $\langle\langle-d,-e,-f,-b\rangle\rangle$; but $q$ is isotropic over $E$ and hence over $E(\sqrt{c(b d e f)}$, so it is hyperbolic over the latter field. That means that $q$ is divisible by $\langle\langle c($ bde $f)\rangle\rangle$ over $E$, as we want.

As a result, all the higher Witt indices of $q$ are even; given the anisotropic parts of $q$ we have observed, it follows that $q$ has splitting pattern $(2,2,2,2)$. (Equivalently, the possible dimensions of the anisotropic part of $q$ over all field extensions of $k$ are $16,12,8,4,0$.)

Finally, we show that $q$ is not divisible by a binary form over $k$. It suffices to show this over the field extension $E=k_{0}(d, e, f, b)((t))$ where $t:=b c d e f$. We can write $q=\langle\langle-d,-e,-f,-b\rangle\rangle-b \operatorname{de} f\langle\langle t\rangle\rangle \otimes\langle 1, d, e, f\rangle$ in the Witt group. If $q$ is divisible by a binary form $\langle\langle u\rangle\rangle$, then $q$ becomes hyperbolic over $E(\sqrt{u})$, from which the properties of Pfister forms imply that $\langle\langle-d,-e,-f,-b\rangle\rangle$ and (consequently) -bdef $\langle\langle t\rangle\rangle \otimes\langle 1, d, e, f\rangle$ become hyperbolic over $E(\sqrt{u})$. But modulo squares, every $u$ in $E^{*}$ is either of the form $c$ or $c t$ for some $c$ in $k_{0}(d, e, f, b)^{*}$. And the passage from $E$ to $E(\sqrt{c t})$ cannot kill $\langle\langle-d,-e,-f,-b\rangle\rangle$, while the 
passage from $E$ to $E(\sqrt{c})$ cannot kill $-b \operatorname{def}\langle\langle t\rangle\rangle \otimes\langle 1, d, e, f\rangle$. Thus $q$ is not divisible by a binary form.

Since Vishik's 16-dimensional form has first Witt index 2, Conjecture 1.1 on ruledness predicts that it is ruled. But since it is not a neighbor of a multiple of a Pfister form, no earlier criterion for ruledness applies. We now prove that this form is in fact ruled. The construction applies to a broader class of forms, including some in arbitrarily large dimensions. In fact, we find that these quadrics are ruled by lines. That has the consequence that any two codimension-1 subquadrics of such a quadric are birational to each other, as in the proof of Theorem 3.1.

TheOREM 7.2. - Let $\rho$ be an $r$-fold Pfister form with $r$ at most 3, $\varphi$ any form, $\sigma$ a nondegenerate subform of dimension at least 2 in $\rho$, and $b$ and $c$ nonzero elements of $k$. Then the form $\varphi \otimes \rho \perp b \sigma \perp c \sigma$ is ruled by lines.

Proof. - We will only write out the proof in the case $\varphi=\langle 1\rangle$. For an arbitrary form $\varphi=\left\langle a_{1}, \ldots, a_{n}\right\rangle$, we just replace the element $r \in \rho$ in the formulas below by a sequence $r_{1}, \ldots, r_{n} \in \rho$.

We can assume (after scaling $\sigma$ ) that $\sigma$ represents 1 . Write $\sigma$ as the orthogonal sum $\sigma=\langle 1\rangle \perp \tau$. Take a standard bilinear multiplication on $\rho$, corresponding to the multiplication on the complex numbers, quaternions, or octonions, so that $\rho(x y)=\rho(x) \rho(y)$. A reference for these algebra structures is [13, Proposition 33.9, Proposition 33.18]. We can assume that $\sigma$ contains the identity element 1 of the algebra as the basis for its subform $\langle 1\rangle$. For $x$ in $\rho$, let $x_{0}$ be the "real part" of $x$, the coefficient of 1 in the decomposition of $\rho$ as the direct sum of $k 1$ and its orthogonal complement, and define the standard involution on $\rho$ by $\bar{x}=2 x_{0} \cdot 1-x$. We automatically have $x \bar{x}=\rho(x) 1, \overline{x y}=\bar{y} \bar{x}$, and $x(\bar{x} y)=(y x) \bar{x}=\rho(x) y$ [13, Proposition 33.9].

Then we define a rational map from the given quadric $\rho \perp b \sigma \perp c \sigma$ to the codimension-1 subquadric $\rho \perp b \sigma \perp c \tau$ by

$$
[r, s, t] \mapsto\left[\left[t\left(t_{0} s-s_{0} t\right)\right] r, s_{0} \rho(t) s-t_{0} \rho(s) t,-\rho(t)\left(t_{0} s-s_{0} t\right)\right] .
$$

Here it is clear that the second and third coordinates are in the linear subspace $\sigma$, and the third coordinate has "real part" equal to 0 so that it lies in the smaller subspace $\tau$.

We have to check that this map sends the quadric $\rho(r)+b \rho(s)+c \rho(t)=0$ into the quadric given by the same formula in $\rho \perp b \sigma \perp c \tau$. Write $\langle$,$\rangle for the bilinear$ form which corresponds to the quadratic form $\rho$, in the sense that $\rho(x)=\langle x, x\rangle$. Since $\rho(x+y)=\rho(x)+2\langle x, y\rangle+\rho(y)$, we find that $2\langle x, y\rangle=x \bar{y}+y \bar{x}=2(x \bar{y})_{0}$ and hence $\langle x, y\rangle=(x \bar{y})_{0}$. We can now begin to compute our quadratic form 
on the output of the above map. The tricky point involves the middle term of the output:

$$
\begin{aligned}
\rho\left(s^{\prime}\right) & =\rho\left(s_{0} \rho(t) s-t_{0} \rho(s) t\right) \\
& =s_{0}^{2} \rho(s) \rho(t)^{2}-2 s_{0} t_{0} \rho(s) \rho(t)\langle s, t\rangle+t_{0}^{2} \rho(s)^{2} \rho(t) \\
& =\rho(s) \rho(t) \rho\left(t_{0} s-s_{0} t\right) .
\end{aligned}
$$

Given that, plus multiplicativity of the form $\rho$, we get a simple formula for the quadratic form applied to the output of the above map:

$$
\rho\left(r^{\prime}\right)+b \rho\left(s^{\prime}\right)+c \rho\left(t^{\prime}\right)=\rho(t) \rho\left(t_{0} s-s_{0} t\right)[\rho(r)+b \rho(s)+c \rho(t)]
$$

which is 0 if $\rho(r)+b \rho(s)+c \rho(t)=0$. Thus we have defined a rational map $f$ from our quadric to a codimension-1 subquadric.

The inclusion from the subquadric $\rho \perp b \sigma \perp c \tau$ into the whole quadric is a section of the above rational map. (Indeed, if $t_{0}=0$, then $t^{2}=-t \bar{t}=-\rho(t) 1$, which implies that the map as written is $s_{0} \rho(t)$ times the identity map.) We can write down another section of this rational map using the transposition map from Lemma 5.1. Explicitly, the restriction of our map to the subquadric $\rho \perp b \tau \perp c \sigma$ defined by $s_{0}=0$ is exactly ( $-t_{0}$ times) the transposition map

$$
[r, s, t] \mapsto[-(t s) r, \rho(s) t, \rho(t) s]
$$

which is a birational map to the base quadric $\rho \perp b \sigma \perp c \tau$. Therefore, the inverse of this map is a section of our rational map, and it is easily checked to be given by the formula

$$
[u, v, w] \mapsto[(w \bar{v}) u, \rho(v) w, \rho(w) v]
$$

Next, we show that the line spanned by these two sections maps into our quadric $q=\rho \perp b \sigma \perp c \sigma$. In other words, for general $[u, v, w]$ in the base quadric $\rho \perp b \sigma \perp c \tau$, we know that $[u, v, w]$ and $[(w \bar{v}) u, \rho(v) w, \rho(w) v]$ lie on $q=0$, and we want to show that the whole line spanned by these two points lies on $q=0$. That follows if we can show that the inner product of these two points is 0 . But we can write that inner product as

$$
\langle u,(w \bar{v}) u\rangle+b\langle v, \rho(v) w\rangle+c\langle w, \rho(w) v\rangle .
$$

Here

$$
\begin{aligned}
\langle u,(w \bar{v}) u\rangle & =[((w \bar{v}) u) \bar{u}]_{0} \\
& =[\rho(u)(w \bar{v})]_{0} \\
& =\rho(u)\langle v, w\rangle .
\end{aligned}
$$


Therefore, the above inner product is

$$
\begin{aligned}
& =(\rho(u)+b \rho(v)+c \rho(w))\langle v, w\rangle \\
& =0,
\end{aligned}
$$

as we want.

Now define a map $X \rightarrow Y \times \mathbf{P}^{1}$ by $[r, s, t] \mapsto\left(f(r, s, t),\left[s_{0}, t_{0}\right]\right)$, where $X$ is the quadric $\rho \perp b \sigma \perp c \sigma, Y$ is the subquadric $\rho \perp b \sigma \perp c \tau$, and $f: X \rightarrow Y$ is the above rational map. The above two sections of $f$ are the fibers over $[0,1]$ and $[1,0]$ in $\mathbf{P}^{1}$. We define a map back from $Y \times \mathbf{P}^{1}$ to $X$ as a suitable linear combination of those two sections:

$$
\begin{aligned}
{[u, v, w],[d, e] } & \mapsto d \rho(w)(u, v, w)+e((w \bar{v}) u, \rho(v) w, \rho(w) v) \\
& =[(d \rho(w)+e(w \bar{v})) u, d \rho(w) v+e \rho(v) w, \rho(w)(d w+e v)] .
\end{aligned}
$$

We will show that these two rational maps are inverses of each other, which will imply that $X$ is ruled by lines, since the map from $Y \times \mathbf{P}^{1}$ to $X$ clearly sends the $\mathbf{P}^{1}$ fibers to lines. It suffices to show that the composition from $X$ to $Y \times \mathbf{P}^{1}$ and back is the identity.

This composition is given by

$$
\begin{array}{r}
{[r, s, t] \mapsto\left[\left[t\left(t_{0} s-s_{0} t\right)\right] r, s_{0} \rho(t) s-t_{0} \rho(s) t,-\rho(t)\left(t_{0} s-s_{0} t\right)\right],\left[s_{0}, t_{0}\right]} \\
\mapsto\left[\left(s_{0} \rho(t)^{2} \rho\left(t_{0} s-s_{0} t\right)-t_{0} \rho(t)\left(t_{0} s-s_{0} t\right)\left(s_{0} \rho(t) \bar{s}-t_{0} \rho(s) \bar{t}\right)\right)\left[\left(t\left(t_{0} s-s_{0} t\right)\right) r\right],\right. \\
s_{0} \rho(t)^{2} \rho\left(t_{0} s-s_{0} t\right)\left(s_{0} \rho(t) s-t_{0} \rho(s) t\right)-t_{0} \rho\left(s_{0} \rho(t) s-t_{0} \rho(s) t\right) \rho(t)\left(t_{0} s-s_{0} t\right), \\
\left.\rho(t)^{2} \rho\left(t_{0} s-s_{0} t\right)\left(-s_{0} \rho(t)\left(t_{0} s-s_{0} t\right)+t_{0}\left(s_{0} \rho(t) s-t_{0} \rho(s) t\right)\right)\right] .
\end{array}
$$

We will show that this composition is equal to $\rho(t)^{2} \rho\left(t_{0} s-s_{0} t\right)\left(s_{0}^{2} \rho(t)-\right.$ $\left.t_{0}^{2} \rho(s)\right)$ times the identity map $[r, s, t]$. This is immediate for the third term (the coefficients of $s$ cancel out). It holds for the second term, using the identity checked earlier that $\rho\left(s_{0} \rho(t) s-t_{0} \rho(s) t\right)=\rho(s) \rho(t) \rho\left(t_{0} s-s_{0} t\right)$. To check it for the first term, in view of the identity $\bar{x}(x r)=\rho(x) r$, it will suffice to show that

$$
s_{0} \rho(t)^{2} \rho\left(t_{0} s-s_{0} t\right)-t_{0} \rho(t)\left(t_{0} s-s_{0} t\right)\left(s_{0} \rho(t) \bar{s}-t_{0} \rho(s) \bar{t}\right)
$$

is equal to $\rho(t)\left(s_{0}^{2} \rho(t)-t_{0}^{2} \rho(s)\right) \overline{t\left(t_{0} s-s_{0} t\right)}$. This is indeed true. Namely, since $t_{0} s-s_{0} t$ has zero "real part", we have $\overline{t\left(t_{0} s-s_{0} t\right)}=-\left(t_{0} s-s_{0} t\right) \bar{t}$, and so the right side of the proposed equality is

$$
=-\rho(t)\left(s_{0}^{2} \rho(t)-t_{0}^{2} \rho(s)\right)\left(t_{0} s \bar{t}-s_{0} \rho(t)\right) .
$$

The left side of the proposed equality, on the other hand, is

$$
\begin{aligned}
& =s_{0} \rho(t)^{2}\left(t_{0}^{2} \rho(s)-2 s_{0} t_{0}\langle s, t\rangle+s_{0}^{2} \rho(t)\right)-t_{0} \rho(t)\left(2 s_{0} t_{0} \rho(s) \rho(t)-s_{0}^{2} \rho(t) t \bar{s}-t_{0}^{2} \rho(s) s \bar{t}\right) \\
& =s_{0} \rho(t)^{2}\left(t_{0}^{2} \rho(s)+s_{0}^{2} \rho(t)\right)-2 s_{0} t_{0}^{2} \rho(s) \rho(t)^{2}-t_{0} \rho(t)\left(s_{0}^{2} \rho(t)-t_{0}^{2} \rho(s)\right) s \bar{t} \\
& =-\rho(t)\left(s_{0}^{2} \rho(t)-t_{0}^{2} \rho(s)\right)\left(t_{0} s \bar{t}-s_{0} \rho(t)\right) .
\end{aligned}
$$


where the second equation uses that $t \bar{s}=2\langle s, t\rangle-s \bar{t}$.

\section{BIBLIOGRAPHY}

[1] H. Анмад \& J. Онм - "Function fields of Pfister neighbors", J. Algebra 178 (1995), p. 653-664.

[2] A. BoReL - Linear algebraic groups, second ed., Graduate Texts in Math., vol. 126, Springer, 1991.

[3] R. Elman, N. A. Karpenko \& A. MerkurJev - The algebraic and geometric theory of quadratic forms, American Mathematical Society Colloquium Publications, vol. 56, Amer. Math. Soc., 2008.

[4] D. W. Hoffmann - "Isotropy of 5-dimensional quadratic forms over the function field of a quadric", in K-theory and algebraic geometry: connections with quadratic forms and division algebras (Santa Barbara, CA, 1992), Proc. Sympos. Pure Math., vol. 58, Amer. Math. Soc., 1995, p. 217225.

[5] _ "Similarity of quadratic forms and half-neighbors", J. Algebra 204 (1998), p. 255-280.

[6] O. T. IzhBoldin - "Quadratic forms with maximal splitting", Algebra $i$ Analiz 9 (1997), p. 51-57.

[7] _ , "Fields of $u$-invariant 9", Ann. of Math. 154 (2001), p. 529-587.

[8] B. KAHN - "A descent problem for quadratic forms", Duke Math. J. 80 (1995), p. 139-155.

[9] N. A. KARPENKO - "Izhboldin's results on stably birational equivalence of quadrics", in Geometric methods in the algebraic theory of quadratic forms, Lecture Notes in Math., vol. 1835, Springer, 2004, p. 151-183.

[10] N. A. Karpenko \& A. MerkurJev - "Essential dimension of quadrics", Invent. Math. 153 (2003), p. 361-372.

[11] M. Knebusch - "Generic splitting of quadratic forms. I", Proc. London Math. Soc. 33 (1976), p. 65-93.

[12] _ "Generic splitting of quadratic forms. II", Proc. London Math. Soc. 34 (1977), p. 1-31.

[13] M.-A. Knus, A. Merkurjev, M. Rost \& J.-P. Tignol - The book of involutions, American Mathematical Society Colloquium Publications, vol. 44, Amer. Math. Soc., 1998.

[14] A. Laghribi - "Formes quadratiques de dimension 6", Math. Nachr. 204 (1999), p. $125-135$.

[15] T. Y. LAM - Introduction to quadratic forms over fields, Graduate Studies in Mathematics, vol. 67, Amer. Math. Soc., 2005. 
[16] S. RousSEY - "Isotropie, corps de fonctions et équivalences birationnelles des formes quadratiques", Ph.D. Thesis, Université de Franche-Comté, 2005.

[17] B. Totaro - "The automorphism group of an affine quadric", Math. Proc. Cambridge Philos. Soc. 143 (2007), p. 1-8.

[18] A. VISHIK - "Motives of quadrics with applications to the theory of quadratic forms", in Geometric methods in the algebraic theory of quadratic forms, Lecture Notes in Math., vol. 1835, Springer, 2004, p. 25-101.

[19] A. R. Wadsworth \& D. B. Shapiro - "On multiples of round and Pfister forms", Math. Z. 157 (1977), p. 53-62. 